\title{
Fundamentación universalista de una lista de capacidades requerida por la justicia global
}

\author{
GustaVo PEREIRA \\ Departamento de Filosofía de la Práctica \\ Facultad de Humanidades y Ciencias de la Educación \\ Universidad de la República (Uruguay) \\ hmodzele@adinet.com.uy
}

\begin{abstract}
Resumen: Tomando como base el marco provisto por una teoría de medios y capacidades, se ofrece una fundamentación universalista de los criterios distributivos y compensatorios propios de la igualdad de capacidades. Para ello se apelará a una fundamentación pragmático trascendental que cuenta con la virtud de conjugar la validez universal con la sensibilidad local. Desde esta perspectiva se critica el uso que hace Nussbaum del equilibrio reflexivo para una fundamentación universalista, aunque se lo incorpora como el momento hermenéutico requerido por la aplicabilidad de la fundamentación universalista.
\end{abstract}

Palabras clave: justicia, igualdad, capacidades, fundamentación

\begin{abstract}
Based on a theory of means and capabilities, this paper offers universalistic foundations to distributive and compensatory criteria, characteristic of the equality of capabilities. In order to do this, certain pragmatic transcendental foundations will be used, which combine universal validity and local sensitivity. From this perspective, Nussbaum's use of reflective equilibrium to reach universalistic grounds is criticised, although the reflective equilibrium is adopted here as the hermeneutic moment which is required by the applicability of such universalistic grounds.

Key words: justice, equality, capabilities, foundations
\end{abstract}

\section{El marco de una teoría de justicia de medios y capacidades}

Probablemente nunca como en nuestra época haya habido tan urgentes requerimientos para una fundamentación universalista en ética. Esto lo pautan tanto los crecientes procesos de globalización económica, como la internacionalización de los problemas ambientales, los conflictos militares y la pobreza. Si bien existen incipientes y más bien tímidas respuestas en distintos ámbitos internacionales, tanto la pobreza como la necesidad de asegurar el desarrollo humano hacen necesario contar con criterios normativos que nos permitan discutir una justicia distributiva mundial. Tal tarea no sólo debe respaldarse con acuerdos fácticos de amplio alcance, sino también con una fundamentación que pueda establecer criterios de validez universal que aseguren 
que no sólo quienes tienen voz y pueden participar en los consensos reales serán tomados en cuenta, sino que lo serán todos, simplemente por su condición de personas. Por otra parte, las exigencias de la diversidad y el multiculturalismo acotan las posibilidades de fundamentación universalista al exigir que la validez universal incorpore el reconocimiento de la particularidad de las diferentes comunidades y culturas.

Para llevar adelante la tarea de presentar las bases de una justicia global, partiré del marco de una propuesta de teoría de justicia distributiva de medios y capacidades que he presentado en otro lugar ( $c f r$. Pereira 2004a y 2004b) y que consiste en la implementación de dos lógicas de criterios distributivos y compensatorios que dependen del estadio de desarrollo de la autonomía del sujeto afectado. Para este enfoque, la autonomía del sujeto se presenta como un concepto estructurante $\mathrm{y}$, al concebirla como un continuo que va desde momentos de desarrollo incipiente hasta la plenitud, podemos introducir un umbral que distingue la autonomía potencial de la plena, estableciendo una lógica distributiva y compensatoria de capacidades para los casos de autonomía potencial, y una lógica de medios para la autonomía plena.

Como criterio normativo que permite diferenciar los estadios de autonomía potencial y plena, he apelado a una lista de capacidades cuyo desarrollo en términos de mínimos posibilita superar el umbral que permite el acceso al estadio de autonomía plena. Estos mínimos, en cuanto que toda persona los puede exigir para poder ser un sujeto autónomo, serían aquello que constituye a la persona y no tiene valor de cambio, sino que vale en sí mismo; por tal razón los he denominado mínimos de dignidad.

Esta lista de capacidades ha sido tomada del desarrollo particular que Nussbaum ha realizado del enfoque de las capacidades, y que no solamente brinda un criterio para establecer el umbral que distingue la autonomía potencial de la plena, sino que también opera como guía normativa para la implementación de políticas públicas. Es justamente en este punto donde surgen algunas dificultades teóricas, porque si bien la necesidad de una lista de capacidades es algo que Sen ha considerado posible, la ha rechazado por razones metodológicas por temor a que una lista sea tan específica que anule la sensibilidad a la variabilidad intercomunitaria que tiene el enfoque. Aquí es donde se centra este trabajo, porque se evaluará el universalismo de Nussbaum, en particular su fundamentación a partir del programa rawlsiano, que enfrenta serias dificultades para superar las limitaciones impuestas por la facticidad de 
los acuerdos. Nuestra intención es presentar una alternativa mejor que la rawlsiana como punto de partida de la fundamentación universalista de una propuesta de justicia distributiva de alcance global. ${ }^{1}$ Este objetivo supone realizar algunos ajustes conceptuales que permitan conjugar la universalidad con la particularidad comunitaria, y para ello apelaremos al programa de fundamentación de la ética del discurso en su parte $\mathrm{B}$.

El recorrido será el siguiente: en primer lugar, se presentarán las características básicas de la igualdad de capacidades, que es el marco en el que se introducirá una lista de capacidades como especificación de la autonomía del sujeto. A la vez se evaluarán las limitaciones de esta perspectiva, para introducir en un segundo momento la parte $\mathrm{B}$ de la fundamentación de la ética del discurso como posible alternativa a tales dificultades.

Por último, se discutirán las virtudes y dificultades que presenta un listado como el de Nussbaum, para luego ofrecer una alternativa que conjugue una fundamentación universalista provista por la ética del discurso y una guía de aplicabilidad estructurada en torno al concepto de equilibrio reflexivo.

\section{La igualdad de capacidades}

El primer momento en esta exposición es presentar la igualdad de capacidades dentro del espectro de la discusión sobre justicia distributiva. Esta perspectiva sostiene que el tratamiento igualitario de las personas se alcanza cuando la distribución o transferencia de recursos se realiza con el objeto de asegurar un conjunto de capacidades que permitan a los afectados alcanzar aquello que es valioso para cada quien, expandiendo de esta forma su libertad real.

La teoría estructurante de la igualdad de capacidades es el enfoque de las capacidades de Sen, aunque a estas alturas existen diversas contribuciones que enriquecen la propuesta. La intención de Sen es proveer un marco normativo que permita realizar la evaluación del bienestar ${ }^{2}$ de las personas. Para ello pretende superar los riesgos subjetivistas que

\footnotetext{
${ }^{1}$ Esta tarea la he desarrollado en forma inicial dentro del marco del constructivismo de Rawls y Dworkin, con el que, si bien tomo distancia en este trabajo, no rompo completamente al resituar el papel del equilibrio reflexivo en el espacio de la aplicabilidad y no en el de la fundamentación, como presentaré en el apartado 8 de este trabajo (cfr. Pereira 2004a, pp. 73-78).

${ }^{2}$ El término bienestar no refiere al uso que se hace en la economía del bienestar, sino a lo que Sen entiende por well-being.
} 
puede tener la búsqueda de una concepción adecuada de este concepto, puesto que es posible que, al categorizar aquello que es valioso para una persona, se incluyan con igual valor todas sus preferencias; esto se da en el caso de las teorías bienestaristas, que asignan igual peso a las preferencias de las personas, cualesquiera sean, lo cual tiene como consecuencia que la preferencia de alguien por realizar un crucero por el Caribe y la de otra persona por satisfacer el hambre sean igualmente relevantes ( $c f r$. Sen 1979, pp. 470-471, y 1994, pp. 146-148). Sen toma distancia de este riesgo y pretende construir un criterio objetivo de bienestar, aunque a diferencia de las propuestas de Rawls y Dworkin, con las que coincide en el rechazo al bienestarismo, tomará también una importante distancia del riesgo de cosificar los medios, ya que es posible que, al concentrarse en ellos como parámetro de justicia, no se perciba la relevancia de la variabilidad interpersonal para las cuestiones de justicia. Para ello, Sen determina un ámbito específico a partir del cual realizar las evaluaciones de justicia: el de las capacidades.

La presentación del concepto de capacidad se puede realizar a partir de la crítica de Sen a la idea de bienes primarios de Rawls, centrada en su rigidez a la hora de ser transformados en bienestar. Esta crítica tiene como base la radical diferencialidad humana que explica el hecho de que una misma cantidad de medios otorgue diferentes cantidades de bienestar a cada persona, dependiendo esto último de las capacidades que tengan estas personas para convertir medios en bienestar.

En consecuencia, la igualdad de medios, que establece como espacio para las comparaciones interpersonales un conjunto de medios objetivos tales como los bienes primarios de Rawls o los recursos de Dworkin, muestra ser insuficiente, mientras que la interpretación de las necesidades en términos de capacidades básicas es lo que permitirá dar cuenta de la igualdad teniendo en cuenta esta variabilidad interpersonal (cfr. Sen 1994, p. 152).

Por lo tanto, para Sen, el concepto de capacidad permite dar cuenta del espacio en el que se puede evaluar la igualdad y realizar las comparaciones interpersonales; esto supone poner el énfasis ya no en las políticas distributivas de medios tales como los bienes primarios o simplemente el ingreso, sino en lo que éstos significan para los seres humanos. La diferencia radical que existe entre las distintas personas vuelve necesario tal movimiento, ya que la conversión de medios varía sustancialmente de una persona a otra, y tal diferencialidad es explicada por el concepto de capacidad; es decir, sólo porque las personas difieren en el desarrollo de sus capacidades es que pueden transformar los mismos medios en logros diferentes.

Diánoia, vol. LI, no. 57 (noviembre 2006). 
Es por esto que Sen sostiene que la vida puede concebirse como un conjunto de funcionamientos o logros; es decir, un conjunto de estados $\mathrm{y}$ acciones en el cual la realización de una persona puede entenderse como el vector de sus funcionamientos, abarcando éstos cosas tales como "estar bien alimentado", "tener buena salud", "participar en la vida de la comunidad", por ejemplo. Los funcionamientos son constitutivos del estado de una persona, y la evaluación del bienestar depende de la evaluación de estos elementos. Por su parte, la idea de capacidad representa las diversas combinaciones de funcionamientos que puede realizar una persona, y de ahí que la capacidad de una persona refleje su libertad para llevar adelante un tipo de vida u otro (cfr. Sen 1995b, pp. 53-54). En función de esto es que las capacidades pueden actualizarse a través de un haz de funcionamientos, y queda a discreción del sujeto la posibilidad de tal actualización; la libertad de una persona, en tal sentido, se encuentra bajo el alcance de esa potencialidad que tienen las capacidades de realizarse por medio de diferentes funcionamientos.

En resumen, en la propuesta de Sen las posiciones individuales no deben ser evaluadas por los recursos que las personas poseen, sino por la libertad que tienen de elegir entre distintas formas de vida. Esta libertad se encuentra representada por la capacidad de una persona para conseguir combinaciones alternativas de funcionamientos.

La proyección del enfoque de las capacidades dentro del marco de la discusión sobre justicia distributiva ha sido enorme. Se ha constituido en los últimos tiempos en un referente ineludible a la hora de evaluar cuestiones tales como la pobreza, el desarrollo o la implementación de políticas públicas. Sin embargo, una de las limitaciones significativas que tiene la igualdad de capacidades es que deja sin responder algunas de las cuestiones más importantes de la justicia social. Esto, en realidad, no puede tomarse como una crítica directa a Sen, puesto que en su trabajo nunca se planteó la tarea de presentar una teoría de la justicia; pero es ineludible considerarlo una vez que pasamos al terreno de la discusión de la justicia distributiva. Es nuestra intención proyectar el enfoque de las capacidades como propuesta que permite asegurar condiciones de mínimos de dignidad a los afectados, desde su aplicación local a una global, y para ello es imprescindible contar con una fundamentación universalista que lo posibilite. Como el enfoque de las capacidades carece de dicho sustento, se apelará a la parte B de la fundamentación de la ética del discurso; es decir, el aspecto de la fundamentación destinado a la aplicabilidad. 


\section{3. Ética del discurso y aplicabilidad}

Para comenzar, es imprescindible recordar que en la propuesta de Apel los presupuestos pragmático-trascendentales de la argumentación, que son irrebasables para todo el que participe en un diálogo, posibilitan en primer lugar la fundamentación de un principio de universalización (U) que adopta el papel de regla de argumentación, por el cual "[t]oda norma válida ha de satisfacer la condición de que las consecuencias y efectos laterales que del seguimiento general de la norma previsiblemente se sigan para la satisfacción de los intereses de cada uno, puedan ser aceptados sin coacción por todos los afectados" (Habermas 1987, p. 68).

También los presupuestos pragmático-trascendentales permiten fundamentar un principio de corresponsabilidad trascendental que afecta a cada argumentante potencial como copartícipe del discurso, y que lo compromete a cooperar en la identificación y solución de los problemas moralmente relevantes que pueden y deben tematizarse en discursos prácticos con arreglo al principio U (cfr. Apel 1993). Esta corresponsabilidad, en cuanto que es inherente a los presupuestos pragmáticotrascendentales de la argumentación, precede trascendentalmente a todas las instituciones sociales, y en función de esto es que Apel sostiene que puede identificarse con la "responsabilidad humana para la creación histórica y cambios necesarios de las instituciones sociales" (Apel 2003, pp. 195-196; cfr. Apel 1993, pp. 27-29). Esto conlleva la corresponsabilidad de dar solución a aquellas situaciones donde los discursos prácticos con arreglo al principio $\mathrm{U}$ no pueden realizarse debido a que los participantes de la interacción, como consecuencia de las circunstancias que los afectan, no pueden intervenir en los discursos. En particular, Apel se refiere a que la aplicación del principio de la ética del discurso solamente puede realizarse donde las relaciones locales de la moralidad y del derecho lo permitan, y en función de ello debe concederse que las normas de contenido básicas de justicia que son filosóficamente fundamentables

no pueden deducirse nunca solamente del principio de la ética del discurso y de su aplicación en un discurso ideal (práctico) de fundamentación de normas. Tienen que poder entenderse también y al mismo tiempo como resultado de la conexión con la tradición ya existente del derecho y de la moralidad de una forma de vida determinada. (Apel 1991, p. 181)

Por todo esto es que la ética del discurso debe complementar su parte A de corte ideal, caracterizada por el compromiso procedimental con 
el principio $\mathrm{U}$, con una parte B comprometida con una mediación entre la racionalidad de la acción estratégico-instrumental y la comunicativoconsensual, es decir, comprometida con la utilización de la racionalidad estratégica orientada a modificar las condiciones que obstaculizan la posibilidad de llegar a soluciones discursivas de los problemas morales. Esto lo realiza a través de dos principios que regulan estas formas de mediación:

a) El primero de tales principios demanda confiar en soluciones discursivas tanto como se pueda, y apelar a tantas estipulaciones estratégicas como sean exigidas a partir de nuestra responsabilidad por las consecuencias esperables de nuestra acción.

b) El segundo principio, por su parte, pretende compensar las implicaciones problemáticas del primero y exige que la mediación entre la racionalidad estratégica y comunicativa, por la que se asegura la utilización de la racionalidad estratégica, se encuentre orientada a modificar las condiciones que obstaculizan la posibilidad de llegar a soluciones discursivas de los problemas morales; es decir, que la motivación de tal mediación se encuentra en el principio regulativo de contribuir a un cambio de la realidad humana conducente a la realización de las condiciones de aplicabilidad de la ética del discurso (cfr. Apel 1993, p. 28).

En esta parte B de la ética del discurso, el principio U se convierte en un valor que puede oficiar como parámetro para un principio teleológico de complementación, que a su vez impone, a través del reconocimiento de la diferencia entre la situación condicionada históricamente de la comunidad real de comunicación y la situación ideal anticipada ya siempre contrafácticamente, la obligación de colaborar en la supresión aproximativa y a largo plazo de la diferencia; en otros términos, estipula el compromiso de realizar la comunidad ideal de comunicación en la real (cfr. Apel 1991, p. 181; 1988, pp. 146-150).

Es preciso remarcar que esta habilitación para la acción estratégica no significa que pueda utilizársela de tal forma que conduzca a la instrumentalización total de las partes enfrentadas, como los enemigos hacen en la guerra o en las negociaciones puramente estratégicas, porque el reconocimiento de la humanidad en la persona de los otros como un fin en sí mismo opera como un límite para la utilización de la acción estratégica. $\mathrm{O}$ más precisamente, esta ética de la responsabilidad que admite la utilización de la acción estratégica o "estrategia contra estrategia" en aquellas situaciones en las que no es posible solucionar 
dialógicamente situaciones conflictivas, al incorporar una complementación teleológica no se transforma en una ética en la que el telos sea subjetivo y, por lo tanto, admita la utilización estratégica de cualquier medio para lograrlo (cfr. Apel 1999, p. 149). Al estar estipulado por la realización del principio $\mathrm{U}$, el telos limita en forma significativa el alcance de la acción estratégica, y por lo tanto restringe los medios aceptables para la realización del fin.

En este punto es preciso ajustar el compromiso de la parte B con la racionalidad estratégica, porque el asegurar la realizabilidad de la parte A no necesariamente debe implicar la utilización de la racionalidad estratégica, sino que es preciso incorporar un criterio de racionalidad prudencial mucho más apropiado para asegurar la aplicabilidad del principio ético a algunos ámbitos como el de la educación, la bioética o la moral cívica ( $c f r$. Cortina 1993, p. 174). Por lo tanto, bien podríamos sostener como lo propio de la parte $\mathrm{B}$ el asegurar las condiciones de realizabilidad sin establecer la utilización exclusiva de un tipo de racionalidad para ello.

Pero más allá de este ajuste que también afecta al ámbito de la justicia distributiva que aquí nos ocupa, igualmente podemos acordar que la parte B de la ética del discurso, al proponer un principio teleológico, puede convertirse en la guía normativa que permita la fundamentación del enfoque de las capacidades y en particular de una posible lista de capacidades. El telos de la comunicación, entendido como la realización de los discursos prácticos en conformidad con el principio $\mathrm{U}$, es el parámetro que permitirá asegurar las condiciones de realización de la autonomía, y de ahí que la lista de capacidades pueda conformarse como respuesta a la pregunta por tales condiciones de realización. A continuación se desarrollará este punto de contacto entre la ética del discurso y el enfoque de las capacidades.

\section{Autonomía como guía contrafáctica}

Como se indicó en el apartado anterior, el telos de la comunicación se convierte en la guía que permitirá asegurar las condiciones de realización de la autonomía que bajo los parámetros de la ética del discurso supone asegurar las condiciones de posibilidad del diálogo.

Con respecto a estas condiciones de posibilidad, estamos obligados a promover su realización, y para ello es necesaria una guía que especifique la autonomía. En tal sentido, el enfoque de las capacidades de Amartya Sen brinda tal guía por tres razones:

Diánoia, vol. LI, no. 57 (noviembre 2006). 
En primer lugar, porque entiende la racionalidad práctica en términos coincidentes con la ética del discurso, es decir, no reducida a una racionalidad de medios a fines, sino incorporando una dimensión moral irreductible. En virtud de esto, un sujeto racional no solamente es capaz de determinar su plan de vida en clave de optimización de la utilidad personal, sino también capaz de reconocer las bases de la cooperación social especificadas a través de la dimensión del compromiso (cfr. Sen 1995a).

También porque supone la libertad y la autonomía como lo propio del hombre, lo que la vuelve una propuesta eleuteronómica que constituye una de las versiones neokantianas contemporáneas más destacadas junto con la ética del discurso y el constructivismo de Rawls. ${ }^{3}$

Por último, el tercer rasgo que permite vincular el enfoque de las capacidades a la ética del discurso se encuentra en la sensibilidad a la diferencia que comparten ambas propuestas. El enfoque de las capacidades, al establecer el tratamiento igualitario respetando la radical diferencialidad intersubjetiva y, por ende, al asegurar el debido reconocimiento de todos los afectados, genera al igual que la ética del discurso las condiciones de ejercicio de la autonomía en términos de reconocimiento recíproco.

En resumen, el enfoque de las capacidades se presenta como una propuesta que posibilita la implementación de una guía normativa para la toma de decisiones y la implantación de medidas de justicia, y por lo tanto como una propuesta que coincidiría con lo requerido por la parte B de la ética del discurso en el campo de la justicia distributiva.

Esta necesidad de construir una guía normativa que permita la evaluación y el diseño de políticas públicas locales y globales requiere la identificación de un conjunto de capacidades que permita, a través de su ejercicio, alcanzar los funcionamientos que posibiliten asegurar la condición de sujetos argumentantes. El criterio igualitario del enfoque de las capacidades operaría como la lógica distributiva y compensatoria que permitiría asegurar la condición de autonomía del sujeto en cuanto sujeto dialógico.

Sen no presenta una lista de capacidades y para ello esgrime una serie de razones metodológicas que serán examinadas en la sección siguiente. Debido a esto y en virtud de la utilidad que una lista con estas

${ }^{3}$ Jesús Conill coincide con esta interpretación al presentar el concepto de libertad en Sen estrechamente ligado a la eleuteronomía en Kant. Quiero también aquí saldar mi deuda con él, quien por primera vez me permitió percibir el kantismo implícito de Sen (cfr. Conill 2004, pp. 190-198). 
características tiene como criterio normativo es que se introducirá la que ha presentado Nussbaum, la cual opera como excelente candidato para nuestros intereses. Se constituye de la siguiente forma:

1. Vida. Ser capaz de vivir una vida completa, no morir prematuramente.

2. Salud. Ser capaz de tener buena salud, incluso salud reproductiva; ser capaz de estar bien alimentado y de tener vivienda adecuada.

3. Integridad física. Ser capaz de moverse libremente, de estar seguro ante ataques de otros, incluyendo violencia sexual en adultos y niños y violencia doméstica; ser capaz de tener oportunidades de satisfacción sexual.

4. Sentidos, imaginación y pensamiento. Ser capaz de usar los sentidos; ser capaz de imaginar, de pensar, y de usar la razón. Todo esto en una forma "verdaderamente humana", es decir, cultivada e informada por una adecuada educación que no se reduzca a la alfabetización y al entrenamiento matemático y científico básico. Ser capaz de usar la imaginación y el pensamiento en conexión con la experimentación y la producción de obras de propia expresión y eventos de propia elección (religiosos, literarios, musicales, etc.). Ser capaz de usar nuestra propia mente en formas protegidas por la libertad de expresión en lo que hace a manifestaciones políticas y artísticas, y a la libertad de cultos. Ser capaz de buscar un significado al sentido de la vida. Ser capaz de evitar el dolor innecesario y de tener experiencias placenteras.

5. Emociones. Ser capaz de experimentar apego a cosas y personas fuera de nosotros mismos; en general, amar, llorar la muerte de alguien, extrañar y sentir gratitud.

6. Razonamiento práctico. Ser capaz de formarse una concepción del bien y reflexionar críticamente acerca de la planificación de su vida.

7. Afiliación:

A) Ser capaz de vivir por y con otros, de reconocer y mostrar consideración por otros seres humanos, de tomar parte en interacciones sociales y familiares. Ser capaz de imaginar la si- 
tuación del otro y tener compasión por tal situación. Tener la capacidad para la justicia y la amistad.

B) Tener las bases sociales del autorrespeto; ser capaz de ser tratado como un ser digno cuyo valor es igual al de los otros. Esto implica una mínima protección contra la discriminación basada en la raza, el sexo, la orientación sexual, la religión, la casta, la etnia o la nacionalidad. Ser capaz de trabajar como un ser humano ejerciendo la razón práctica y participando en relaciones significativas de reconocimiento mutuo con otros trabajadores.

8. Otras especies. Ser capaz de sentir consideración en relación con animales, plantas y la naturaleza.

9. Juego. Tener sentido del humor, ser capaz de jugar y de disfrutar actividades recreativas.

10. Control sobre el propio entorno.

A. Político. Ser capaz de participar efectivamente en las elecciones que gobiernan nuestra propia vida; tener derecho a la participación política, a la protección de la libertad de expresión y asociación.

B. Material. Ser capaz de tener propiedades, no sólo formalmente, sino en términos de oportunidades reales; tener derechos de propiedad sobre iguales bases que otros; tener el derecho a buscar sobre iguales bases que otros (Nussbaum, 2000a, pp. 78-80). ${ }^{4}$

Nussbaum también introduce la idea de un umbral interno a cada capacidad, que delimitaría el mínimo nivel de desarrollo de capacidades necesario para adquirir la condición de autonomía del sujeto. A estos mínimos los he denominado mínimos de dignidad, porque son aquello a lo que se tiene derecho sólo por tener la condición de persona.

A su vez, este umbral posibilita la introducción de la distinción conceptual, ya señalada en la primera sección de este artículo, entre autonomía potencial y autonomía plena fundada en diferencias de desarrollo en el conjunto de capacidades básicas (cfr. Pereira 2004a y 2004b). Esta idea de potencialidad, determinada por un bajo nivel en el desarrollo de capacidades, estaría indicando que si los afectados fuesen sometidos a políticas compensatorias que apuntaran al desarrollo

${ }^{4}$ David Crocker también presenta un conjunto tentativo que coincidiría a grandes rasgos con el de Nussbaum (cfr. Crocker 1998). 
de capacidades, necesariamente alcanzarían el estado de autonomía plena. Esta relación entre potencialidad y plenitud genera el deber de que las políticas públicas sean orientadas a superar esa brecha. La razón para esto último se asienta en que un desarrollo mínimo de capacidades básicas se presenta como condición de posibilidad de toda situación de diálogo y, a su vez, en el ejercicio de su condición de argumentante se encuentra lo propio de un sujeto autónomo.

Es necesario indicar que la lista es una lista abierta y general, que permite variaciones en su aplicación, tanto por las múltiples especificaciones de cada uno de sus componentes como por las distintas sociedades en las que se aplique. Esta variabilidad no afecta al conjunto de capacidades básicas, sino que lo especifica, por lo que la variabilidad del umbral dependerá de cómo cada sociedad provea los mínimos de dignidad, siendo justamente esta variabilidad la que permite asegurar que las bases del reconocimiento sean aseguradas transculturalmente. Asimismo, el mínimo de capacidades básicas podrá ser desarrollado en la forma que cada sociedad considere pertinente, por lo que la expansión de la libertad se orientaría en función de la diversidad de cada sociedad y de sus características propias en lo que hace a visiones comprehensivas, pero siempre asegurando universalmente el mínimo que permite contar con sujetos autónomos. Esta característica de exigibilidad universalista de un conjunto de mínimos de justicia y de su aplicabilidad en consonancia con las demandas locales confirma nuestra propuesta de presentar la igualdad de capacidades especificada —en este caso, a través del particular desarrollo que realiza Nussbaumcomo el mejor criterio distributivo y compensatorio para todos aquellos sujetos que se encuentran en una situación de autonomía potencial. Por ello es que la igualdad de capacidades en esta versión constituye la parte más básica de la propuesta de justicia distributiva de medios y capacidades que estamos desarrollando como la requerida por la parte $\mathrm{B}$ de la ética del discurso y fuertemente sustentada en sus supuestos del sujeto.

Por último, si bien este conjunto de capacidades básicas, que son condición de posibilidad de un sujeto autónomo en cuanto sujeto argumentante de reconocimiento recíproco, nos permite contar con una guía que posibilita la evaluación de las políticas de justicia global, es preciso detenerse en algunas de las dificultades conceptuales que tal lista ha generado, tanto dentro del propio enfoque de las capacidades como en la discusión con otros posibles candidatos. A continuación se examinarán los reparos de Sen a la presentación de una lista de capacidades, la exclusión de la distinción conceptual de agencia y bienestar 
por parte de Nussbaum, y en última instancia la función del equilibrio reflexivo en su estrategia de fundamentación universalista.

\section{Dificultades conceptuales de la lista de capacidades}

\section{1. La crítica de Sen}

La propuesta de Nussbaum se encuentra dentro del programa del enfoque de las capacidades de Amartya Sen, y si bien este último admite la posibilidad de construir una lista de capacidades dentro de ese paradigma, también es necesario recordar que toma franca distancia de todo intento de presentar una lista concreta, al sostener la imposibilidad metodológica de una ordenación completa. Según Sen, su opción por el uso de la alternativa de una ordenación parcial se sustenta en dos razones; la primera de ellas es que la idea de desigualdad presenta cierta ambigüedad, lo que determinaría que un intento de ordenación completa, al basarse en un concepto ambiguo, fuese erróneo. La segunda razón es que "aunque no fuera un error buscar una ordenación completa, en la práctica podemos no ser capaces de identificarla" (Sen 1995b, pp. 62-63). Pero si bien tanto la posibilidad del error como la de la propia falibilidad desaconsejan una ordenación completa, Sen sostiene que es perfectamente posible realizar un ordenamiento parcial que habilite la jerarquización de algunas situaciones sobre otras.

En concordancia con estas razones metodológicas, Sen rechaza la lista de Nussbaum porque, a pesar de que él reconoce que las capacidades tienen una variación intercomunitaria considerablemente menor que la de los requerimientos de medios para su ejercicio, considera que una lista fija con pretensiones de universalidad es tan específica que reduce las posibilidades de ajuste local, socavando la sensibilidad a la variación intercomunitaria, que es una de las consecuencias más valiosas de la incompletitud del ordenamiento. De hecho, tanto la potencialidad como la utilidad del enfoque, y debemos agregar su conexión con un programa de fundamentación como el de la ética del discurso, se encuentran respaldados en una importante distinción conceptual entre la generalidad del enfoque de las capacidades y la particularidad de un listado específico de funcionamientos que apunta a salvaguardar dicha variación intercomunitaria (Sen 1993, p. 47).

Sin embargo, es necesario reconocer en defensa de Nussbaum que sus trabajos tienen como principal objetivo asegurar la sensibilidad a la variabilidad intercomunitaria; esto último es inherente al concepto de capacidad en la medida en que guarda una relación de potencialidad con respecto a la realizabilidad de los funcionamientos, siendo lo 
que posibilita que un mismo conjunto de capacidades básicas pueda ser especificado en forma sustancialmente diferente en función de las condiciones locales, variando tanto el peso interno de cada capacidad como el nivel de desarrollo de las mismas. Es más, este desarrollo particular del enfoque de las capacidades que realiza Nussbaum permite superar cualquier sospecha de perfeccionismo que se le pueda imputar desde una perspectiva liberal (Rawls 1996, pp. 216-220), ya que aquello que debe ser objeto de las políticas públicas no es un listado de funcionamientos que especificarían cierta idea de buena vida, sino el conjunto de capacidades que colocan a todas las personas en condiciones de poder elegir y realizar un plan de vida (Nussbaum 2000a, pp. 87-90) ${ }^{5}$.

Una vez que el concepto de capacidad adquiere el carácter transcultural y transcomunitario que Nussbaum le impone y se especifica comunitaria y culturalmente a través de un conjunto de funcionamientos, la crítica de Sen se diluye. De hecho, lo que debería criticarse a la lista como cuestionamiento a sus bases de fundamentación, no es el riesgo de ser ciega a la variabilidad cultural, sino su caracterización de la persona o del sujeto que es digno de tratamiento igualitario. Sen no toca este punto porque teme caer en una fundamentación metafísica, pero lejos está la fundamentación de Nussbaum de remitir a una esencia humana, como sería el caso del aristotelismo tradicional, sino que su aristotelismo, fuertemente situado en la modernidad a través de la idea de persona moral de Rawls, es un aristotelismo político y no metafísico, sustentado en una fundamentación que apela a la noción de equilibrio reflexivo como base de tal listado. ${ }^{6}$ Esto último constituye el punto que considero más débil de la propuesta de Nussbaum; pero antes de discutir si la opción por el equilibrio reflexivo es apropiada

${ }^{5}$ Como ejemplo fallido es de destacar el caso de Sabina Alkire (cfr. 2002), quien presenta una lista alternativa a la de Nussbaum y no logra superar esta dificultad. La razón para ello es que se basa en la concepción de sujeto de Finnis, y al introducir éste cierta idea de naturaleza humana no logra superar los cuestionamientos que podrían hacerse desde una perspectiva liberal. Es de destacar que para Finnis, y en esta crítica también coincide Nussbaum (cfr. 2000a, p. 95, nota 110), una comunidad hedonista y atea estaría basada en algo así como una comprensión moral distorsionada ( $c f r$. Finnis 1999), y esto es inaceptable desde toda perspectiva que pretenda asegurar el tratamiento igualitario.

${ }^{6} \mathrm{Si}$ bien este aristotelismo ha sido cuestionado por ser excesivamente moderno, no incurre en una fundamentación metafísica. Puede decirse que la carga de la prueba que debe enfrentar es la de cuán aristotélica es su fundamentación al asumir la idea de persona moral como estructurante (cfr. Mulgan 2000, pp. 85-99; Conill 2004, pp. 182-186; Nussbaum 2000b, pp. 103-116).

Diánoia, vol. LI, no. 57 (noviembre 2006). 
para sus intenciones de fundamentación universalista, quiero considerar su rechazo de una distinción conceptual utilizada por Sen, la que se da entre agencia y bienestar, que a mi entender también debilita considerablemente su planteamiento.

\subsection{Agencia y bienestar: el camino kantiano de mejorar la lista}

Nussbaum explícitamente rechaza la distinción de Sen entre agencia y bienestar. En su opinión, tanto ésta como la de libertad y realizaciones se encuentran capturadas como aspectos de la distinción más básica entre capacidad y funcionamientos (Nussbaum 2000a, p. 14). Al respecto sostendré que si bien el par libertad y realizaciones puede ser remitido sin pérdida al par capacidad/funcionamientos, no sucede lo mismo con agencia/bienestar, que es una distinción crucial a la hora de establecer el peso diferencial que deben tener las capacidades a la hora de establecer ordenamientos específicos de funcionamientos. Lo que quiero sustentar es que la distinción agencia/bienestar atraviesa el par capacidad/funcionamientos, de tal forma que no puede ser asimilada exitosamente a esta última sin una pérdida explicativa considerable. Por otra parte, el adoptarla posibilita contar con un instrumento más apropiado para guiar normativamente el diseño de políticas públicas.

Para sustentar esta interpretación, voy a remitirme al supuesto de sujeto racional que presenta Sen. Éste se basa en la incorporación de una dimensión moral de comportamiento racional, irreductible a la lógica de medios a fines y que se denomina compromiso. Las acciones basadas en el compromiso tienen como característica distintiva una fuerte ruptura de la relación de determinación entre elección personal y el bienestar que se presenta en las otras dimensiones del comportamiento racional, justificando acciones que incluso pueden ir en contra del propio bienestar (cfr. Sen 1995a, pp. 90 y ss; Pereira 2004b, pp. 12-14). En este punto queda de manifiesto la importante coincidencia de Sen con el pensamiento kantiano que indicamos antes, ya que la acción determinada por su dimensión del compromiso coincidiría con el actuar bajo la fórmula del imperativo categórico, el cual obliga en forma incondicionada y exige actuar de tal modo que el otro siempre sea considerado como un fin y nunca solamente como un medio; un fin que no tiene valor relativo o precio, sino que es un fin en sí mismo, que tiene valor interno, es decir, dignidad (cfr. Kant 1981, pp. 84, 92-92).

Esta doble dimensión del comportamiento racional en Sen, en la que se da por una parte una lógica de medios a fines y de identidad entre elección y bienestar, y por otra una lógica basada en lo que tiene valor 
en sí, con lo que se rompe la identidad entre elección y bienestar, es correlativa con su distinción entre agencia y bienestar, ya que la acción de medios a fines que orienta la conducta basada en la optimización del propio provecho sería lo propio de la faceta de bienestar, mientras que la acción basada en el compromiso sería lo propio de la faceta de agencia.

En consonancia con esta distinción entre facetas de bienestar y de agente, es posible establecer la distinción entre libertad de bienestar y libertad de agencia. La libertad de bienestar se centra en la capacidad de una persona para disponer de varios tipos de funcionamientos y gozar de las correspondientes consecuciones de bienestar. La libertad de ser agente, por su parte, es un concepto más amplio de libertad y se refiere a lo que la persona es libre de hacer y conseguir en la búsqueda de metas o valores que considere importantes. Esta idea de libertad se encuentra basada en la faceta de agente de la persona, la cual es incomprensible si no se tiene en cuenta la concepción del bien que estructura las metas y los valores de esta persona (cfr. Sen 1997, p. 86).

La importancia que tiene esta distinción entre libertad de bienestar y de ser agente, y la irreductibilidad de la última a la primera, nos coloca ante el hecho de que estas facetas requieren tratamientos diferentes en la evaluación moral. La faceta de bienestar es relevante en la evaluación de cuán provechosa es la vida de alguien, mientras que la faceta de agente es relevante en la evaluación de cómo lleva alguien a cabo su concepción del bien. La capacidad de una persona para llevar a cabo su concepción del bien no tiene por qué ser provechosa para ella; es más, puede atentar contra su bienestar, y en tal caso la pérdida de bienestar se encuentra justificada por la ganancia en la libertad de ser agente, pudiéndose hablar de ganancia neta en este tipo de libertad. A su vez, Sen pone de manifiesto la profunda diferencia entre las perspectivas de ser agente y de bienestar, ya que si bien en algunas circunstancias la libertad de ser agente puede coincidir con la perspectiva de bienestar, de ninguna manera puede afirmarse que la primera subsuma a la segunda.

Desde la perspectiva de ser agente, la persona es considerada como alguien que juzga y actúa, mientras que desde la perspectiva de bienestar se lo considera como un beneficiario cuyos intereses y ganancias han de ser considerados. De acuerdo con esto, la faceta de bienestar es cardinal en algunas circunstancias, como en la planificación de políticas de cobertura sanitaria básica, mientras que en otras la faceta de ser agente y la propia responsabilidad hacia los demás son determinantes; por ejemplo en acciones de la vida comunitaria. En esto último reside 
la importancia de la distinción, ya que la incorporación del hecho de que circunstancias diferentes determinarán qué faceta se priorizará en una política pública y también qué prioridad se dará a unas capacidades sobre otras, permite una mayor precisión en la aplicación de la guía normativa que pretende ser una lista de capacidades. Por ejemplo, en el primer caso que se indicó, el de una política sanitaria, si bien las dos facetas son relevantes, la de bienestar desempeña un papel preponderante, al igual que si pensamos en una política que ataque la deficiencia nutricional de una población. Por otra parte, si el objetivo es diseñar una política pública que fortalezca la participación ciudadana en decisiones comunitarias, es la faceta de agencia la que primará.

De acuerdo con esto último, lo que se pierde en la lista de Nussbaum al excluir la distinción agencia/bienestar es la posibilidad de establecer reglas de prioridad entre las capacidades que faciliten el ordenamiento de los funcionamientos que se han de asegurar; ésta es una herramienta primordial que no es reductible a la distinción capacidad/funcionamientos, sino que, por el contrario, afecta transversalmente estos conceptos y, por lo tanto, es un elemento ineludible a la hora de utilizar la guía normativa de la lista de capacidades. En consecuencia, es posible distinguir dentro del listado y en ciertas circunstancias capacidades más propicias para responder a las demandas de bienestar que otras; esto también se manifiesta en los funcionamientos que se pretenda asegurar, por lo que esta distinción se convierte en una herramienta imprescindible para diseñar con mayor precisión políticas públicas.

Por último, es importante destacar que, en este caso, la precisión no es asimilable a un ideal cuantificador-cosificador, sino que surge del peso que tendrá en cada caso particular cada capacidad, y eso establece una lógica de prioridad para el ordenamiento, de tal manera que si estamos ante una situación de emergencia sanitaria o una hambruna, es indudable que habrá que priorizar las capacidades que permitan potenciar la faceta de bienestar, y el ordenamiento las hará pesar de forma más significativa que, por ejemplo, las del razonamiento práctico que afectan principalmente a la faceta de ser agente. Esto no significa que las dos facetas no interactúen y que no sea necesario propiciar un desarrollo global de capacidades que tenga como objetivo final expandir tanto la libertad de bienestar como la de agencia, sino que pretende enfatizar la relevancia que tiene esta búsqueda de la precisión para la obtención de resultados positivos a corto plazo en el diseño de políticas sociales en un contexto de escasez relativa de medios. En los países más pobres, la mayor precisión que provee la distinción entre agencia 
y bienestar se convierte en un requerimiento indispensable, ya que no obtener buenos resultados en la implementación de una política social significa que habrá recursos mal invertidos, y en consecuencia habrá menos disponibilidad para alguien que en verdad los necesita. Por lo tanto, toda lista de capacidades que tenga la intención de operar como guía normativa para el diseño de políticas públicas no podrá menos que contar con el complemento de esta distinción a la hora de la aplicabilidad del enfoque.

\subsection{El rol del equilibrio reflexivo}

En este último punto se considerará si una fundamentación como la que presenta Nussbaum es la más apropiada para su lista de capacidades universales. Para ello nos preguntaremos si una posición como la rawlsiana, que en la problemática de Liberalismo político toma una explícita y significativa distancia de una teoría universalista de justicia para limitarse a un liberalismo referido a las sociedades democráticas, puede oficiar como el mejor soporte para una lista universalista de capacidades. La tarea que emprende Nussbaum se encuentra fuertemente urgida por la realidad de un mundo crecientemente globalizado y que demanda criterios de justicia que puedan aplicarse más allá de la propia sociedad para situarse en el contexto mundial, lo que a su vez exige la fundación de una ética global. Pero, para lograr este objetivo, sostendré que el apelar al concepto de equilibrio reflexivo, que desarrollaré más adelante, es francamente insuficiente, y en tal sentido se presenta como mejor alternativa la fundamentación pragmáticotrascendental de la ética del discurso, que ofrece en su parte B una guía normativa para un proyecto como el de Nussbaum. Sin embargo, esto no significa descartar totalmente al equilibrio reflexivo, porque puede cumplir la función de suplir el déficit hermenéutico que tiene la fundamentación universalista de la ética del discurso a la hora de la aplicabilidad.

Recordemos que bajo una interpretación pragmático-trascendental, una lista de capacidades especificaría condiciones posibilitantes de la parte A de la ética del discurso, o más claramente cuáles son las condiciones de realizabilidad de un sujeto argumentante. Esta lista se conformaría a partir de la pregunta de cuáles son las capacidades que le permiten a alguien tomar parte en un diálogo, mientras que la pregunta acerca de cuál es el desarrollo mínimo de estas capacidades para tomar parte en un diálogo especificaría el umbral de dignidad. En cuanto a las posibles listas, la de Nussbaum se presenta como un fuerte candidato y 
parece considerablemente más ventajosa que otras propuestas, porque las características de universalidad, de no especificidad de la lista y la incorporación de un umbral de dignidad, permiten responder de buena forma a las exigencias universalistas y a las especificaciones comunitarias de nuestras sociedades contemporáneas. Por último, cabe recordar que la apertura de la lista de Nussbaum, en la que ella insiste una y otra vez, evita toda posible rigidez en su construcción. Pero, además de estas virtudes, lo central en la perspectiva que quiero defender es que la conformación de la lista siempre estará disponible a una mejor interpretación guiada por la fundamentación pragmático-trascendental, que al considerar las exigencias de fundamentación universalista de una justicia global se presenta como una alternativa considerablemente más sólida que el equilibrio reflexivo rawlsiano propuesto por Nussbaum (cfr. 2000a, pp. 101-103).

Para comenzar con una evaluación más precisa de los alcances del equilibrio reflexivo como candidato a fundamentar una lista universal de capacidades, debemos recordar que el equilibrio se alcanza cuando el filósofo se cerciora de que las ideas normativas reconstruidas se ajustan a los juicios reflexivos de los afectados por tales ideas (cfr. Rawls 1979, p. 38), o también cuando una concepción "consigue articular nuestras convicciones más firmes acerca de la justicia política, a todos los niveles de generalidad, después del examen debido, y una vez hechos los reajustes y las revisiones que parecen obligados" (Rawls 1996, p. 59). De esta forma, una concepción de justicia remite a la tradición democrática de la propia sociedad, lo que hace que las ideas normativas postuladas, si bien cuentan con un fuerte anclaje empírico, difícilmente pueden superar los límites que impone la propia tradición, y en función de ello la posibilidad de fundamentación universalista es socavada. El giro hermenéutico rawlsiano, para decirlo con la terminología de McCarthy (cfr. 1994, p. 61), debilita sustancialmente toda intención de fundamentación universalista de justicia global al ser contrapesada por la contextualización que impone toda interpretación hermenéutica y que establece la prioridad de la propia tradición cultural sobre cualquier principio independiente a través del preconocimiento del propio mundo de la vida.

Estas consecuencias hacen que Nussbaum se vea atrapada en la red de algunas críticas que, además de considerar el momento hermenéutico de la reconstrucción de la propia tradición, demandan un criterio que permita discriminar entre diferentes interpretaciones, de tal forma que se marque el progreso interpretativo. Sin embargo, como ya se adelantó, esta debilidad no significa anular la metodología del equilibrio 
reflexivo, sino acotar su alcance. Una fundamentación procedimentalista como la de la ética del discurso para poder alcanzar una validación universal debe abstraerse de contenidos sustanciales, tradiciones o supuestos pragmáticos; en consecuencia, esta fundamentación tiene como contracara un fuerte déficit hermenéutico que la carga con importantes dificultades a la hora de responder a las demandas de la aplicabilidad (cfr. Conill 2003, pp. 124-125; Cortina 1993, pp. 176-177). Si bien este problema pretende ser solucionado por la parte B presentada por Apel, tal alternativa no es suficiente y puede ser complementada por una metodología eminentemente hermenéutica como el equilibrio reflexivo rawlsiano, que remitiendo a la propia tradición, cultura, mundo de la vida, contexto histórico y vital de las distintas comunidades, se convierte en una herramienta clave para una ética global, pero ya no en lo que toca a su fundamentación, sino a su aplicación. De hecho, esto último es lo que realiza Nussbaum, aunque lo que pretenda con ello sea zanjar el problema de la fundamentación. ${ }^{7}$

Lo que podría denominarse el bloqueo hermenéutico del equilibrio reflexivo como posible fundamentación para una justicia global se asienta en que no solamente debe cargar con la primacía de la propia tradición, sino que también, como el propio Rawls afirma en contraposición al intuicionismo, el equilibrio reflexivo procede indefinidamente a causa de la permanente revisabilidad que lo afecta (cfr. Rawls 1996, pp. 126127). ${ }^{8}$ Esta restitución al interpretandum de la posibilidad de siempre ser mejor comprendido respalda una asimetría entre interpretandum e intérprete en favor del primero, lo que tiene por consecuencia la disolución de toda capacidad crítico-reflexiva, ya que, como se había señalado, no hay un criterio desde donde fundar esta capacidad crítica. Ante esto, el modelo propuesto por Apel, si bien se sitúa dentro de la tradición hermenéutica, introduce una idea regulativa para el progreso cognoscitivo que denomina norma ética fundamental, que exige la autoafirmación de la comunidad real de comunicación y la realización de la comunidad ideal de comunicación.

La comunidad ideal de comunicación apeliana propone una "idea regulativa" para evaluar las sociedades actuales por lo próximas o alejadas que estén de dicha idea (cfr. Apel 1985, pp. 203-205). En ella se

\footnotetext{
${ }^{7}$ Esta complementación de la parte B de la ética del discurso por el equilibrio reflexivo estaría en concordancia con la propuesta de Kuhlman, quien explícitamente introduce al equilibrio reflexivo como mediador entre la fundamentación del principio moral y las propuesta de contenido moral (cfr. Kuhlmann 1996).

${ }^{8}$ No sólo Rawls se ve afectado por esta dificultad, sino también Dworkin, quien incorpora supuestos metodológicos similares (cfr. Pereira 2003).
}

Diánoia, vol. LI, no. 57 (noviembre 2006). 
establecen como características distintivas la exclusión de la desfiguración de la comunicación, se aseguran simétricamente las oportunidades de elegir y realizar actos de habla, a la vez que se garantiza la intercambiabilidad de los roles del diálogo. Por referencia a la comunidad ideal es posible criticar el hecho de que en la vida real no haya auténticos diálogos. Quien argumenta presupone la comunidad ideal de comunicación como posibilidad de la sociedad real, y por ello ha de postular moralmente la disolución histórica de la contradicción dialéctica entre la comunidad ideal y la real (cfr. Apel 1985, pp. 409).

De lo anterior es posible afirmar que la metodología del equilibrio reflexivo, al no ofrecer un criterio que permita identificar el progreso interpretativo, queda confinada al contextualismo que impone la vigencia empírica de la tradición de la sociedad o sociedades en cuestión, y que socava las posibilidades de fundamentar una propuesta universalista, ya que la mera facticidad opacaría las situaciones de quienes no tienen voz o tienen una voz muy débil, básicamente los pobres, las minorías étnicas y la mujer y su sujeción, entre otros. Una fundamentación universalista requiere por su parte apelar a criterios que vayan más allá de la facticidad, y en tal sentido la fundamentación de la ética del discurso se presenta como un candidato más sólido.

Puede afirmarse en este punto una doble necesidad para una justicia global; por un lado, la necesidad de que una metodología como el equilibrio reflexivo cuente con un parámetro universalista que le permita superar el bloqueo hermenéutico, pero también la necesidad de que un principio universalista pueda tener la suficiente densidad hermenéutica como para dar respuesta a cuestiones de aplicabilidad. En tal sentido, la parte B de la ética del discurso puede valerse del equilibrio reflexivo para reconstruir el significado que, por ejemplo, asume para una sociedad la lista de capacidades o el conjunto de bienes por distribuir, o cómo deberían especificarse los criterios distributivos. La debilidad de Nussbaum no está en apelar al equilibrio reflexivo, sino en el papel que le pretende hacer cumplir. Bajo objetivos menos ambiciosos, esta metodología es de suma utilidad para dar respuesta a cuestiones de aplicabilidad, como efectivamente lo ejemplifica Nussbaum (2000a, pp. 101-105); pero una vez que pasamos de su función en el nivel de aplicabilidad al de fundamentación de una justicia global universalista, quedan de manifiesto sus debilidades.

Estas cuestiones refieren a dos momentos que toda teoría de justicia global debe recorrer: el de la fundamentación universalista y el de la aplicabilidad con su carga hermenéutica. Discriminar dos procedimien- 
tos con potencias diferenciales para cada caso, como hemos hecho más arriba, parece ser la estrategia más apropiada.

\section{Conclusión}

$\mathrm{Al}$ inicio de este trabajo señalamos una propuesta de justicia distributiva de medios y capacidades que operó como marco para esta reflexión. Para esta perspectiva y también para todas aquellas que utilicen criterios distributivos y compensatorios propios de la igualdad de capacidades se vuelve necesario contar con una fundamentación de una lista de capacidades que pueda operar como guía normativa tanto a nivel local como global.

En tal sentido se ha presentado una fundamentación que conjuga la validez universal con las exigencias de aplicabilidad. Esto se ha logrado a través de la conexión propuesta entre el enfoque de las capacidades y la ética del discurso en la versión de Apel, cuya parte B, al operar como un principio teleológico, permite realizar una fundamentación universalista del enfoque de las capacidades mucho más sólida que la que Nussbaum pretende a través del equilibrio reflexivo.

Por último, no eliminamos el equilibrio reflexivo, sino que lo resituamos en el plano de la aplicabilidad para lograr una complementación para esta propuesta de justicia distributiva entre la parte B de la ética del discurso y la metodología del equilibrio reflexivo. Con esto se pretende suplir tanto el déficit de fundamentación universalista del equilibrio reflexivo, como el déficit hermenéutico para la aplicabilidad de la fundamentación pragmático-trascendental.

\section{BIBLIOGRAFÍA}

Alkire, Sabina, 2002, "Dimensions of Human Development", World Development, vol. 30, no. 2, febrero de 2002, pp. 181-205.

Apel, Karl-Otto, 2003, "Globalización y necesidad de una ética universal", en A. Cortina y D. García-Marzá, Razón pública y éticas aplicadas, Tecnos, Madrid, pp. 191-218.

—_, 1999, Filosofia primera, avui i ètica del discurs, Eumo-Universistat de Girona, Girona.

— 1993 , "How to Ground a Universalistic Ethics of Co-Responsibility for the Effects of Collective Actions and Activities", Philosophica, vol. 52, no. 2, pp. 9-29.

_ 1991 , "La ética del discurso como ética de la responsabilidad. Una transformación posmetafísica de la ética de Kant", en K.-O. Apel, Teoría de la

Diánoia, vol. LI, no. 57 (noviembre 2006). 
verdad y ética del discurso, trad. Norberto Smilg, Paidós/I.C.E.-U.A.B., Barcelona, pp. 147-184.

Apel, Karl-Otto, 1988, Diskurs and Verantwortung, Suhrkamp, Fráncfort del Meno.

— 1985, La transformación de la filosofía, Taurus, Madrid, vol. 2.

Conill Jesús, 2004, Horizontes de economía ética, Tecnos, Madrid.

—_, 2003, "El carácter hermenéutico y deliberativo de las éticas aplicadas", en Adela Cortina y Domingo García-Marzá (comps.), Razón pública y éticas aplicadas, Madrid, Tecnos, pp. 121-142.

Cortina, Adela,1993, Ética aplicada y democracia radical, Madrid, Tecnos.

Crocker David, 1998, "Consumption, Well-Being and Capability", en D. Crocker y T. Linden (comps.), Ethics of Consumption. The Good Life, Justice and Global Stewardship, Rowman and Littlefield Publishers, Nueva York, pp. 336-390.

Finnis, John, 1999, "Natural Law and Ethics of Discourse", Ratio Juris, vol. 12, no. 4, pp. 354-373.

Habermas, Jürgen, 1987, Escritos sobre moralidad y eticidad, Paidós/I.C.E./ U.A.B, Barcelona.

Kant, Immanuel, 1981, La fundamentación de la metafísica de las costumbres, Espasa-Calpe, Madrid.

Kuhlmann, Wolfgang, 1996, "Diskursethik und die neuere Medizin. Anwendungsprobleme der Ethick bei wissenchafttlichen Innovationen", en Jan P. Beckmann (ed.), Fragen und Probleme einer medizinischen Ethik, De Gruyter, Berlín, pp. 94-117.

McCarthy, Thomas, 1994, "Kantian Constructivism and Reconstructivism: Rawls and Habermas in Dialogue", Ethics, vol. 105, no. 4, pp. 44-63.

Mulgan, Richard, 2000, "Was Aristotle an "Aristotelian Social Democrat?", Ethics, vol. 111, no. 1, octubre de 2000, pp. 79-101.

Nussbaum, M., 2000a, Women and Human Development, Cambridge University Press, Nueva York.

—, $2000 \mathrm{~b}$, "Aristotle, Politics, and Human Capabilities: A Response to Antony, Arneson, Charlesworth, and Mulgan", Ethics, vol. 111, no. 1, octubre de 2000, pp. 102-140.

Pereira, Gustavo, 2004a, Medios capacidades y justicia distributiva, Instituto de Investigaciones Filosóficas-UnAM, México.

—_, 2004b, "Justicia distributiva: medios y capacidades", Diánoia, vol. 49, no. 53, pp. 3-32.

Rawls, John, 1996, El liberalismo político, trad. Antoni Domenech, Crítica, Barcelona.

—_, 1979, Teoría de la justicia, trad. María Dolores González, Fondo de Cultura Económica, México.

Sen, Amartya, 1997, "El bienestar, la condición de ser agente y la libertad. Conferencias 'Dewey' de 1984", en Bienestar, justicia y mercado, trad. Damián Salcedo, Paidós, Barcelona, pp. 38-108. 
1995a, "Rational Fools: A Critique of the Behavioural Foundations of Economic Theory", en A. Sen, Nueva economía del bienestar. Escritos seleccionados, Universitat de València, Valencia, pp. 83-102.

Sen, Amartya, 1995b, Nuevo examen de la desigualdad, trad. Ana María Bravo, Alianza, Madrid.

__, 1994, “ilgualdad de qué?”, en Libertad, igualdad y derecho, trad. G. Valverde Gefall, Planeta-Agostini, Barcelona, 1994, pp. 133-156.

— , 1993, "Capability and Well-Being", en A. Sen y M. Nussbaum, The Quality of Life, Clarendon Press, Oxford, pp. 30-53.

, 1979, "Utilitarianism and Welfarism", The Journal of Philosophy, vol. 76, no. 9, pp. 463-489.

Recibido el 6 de junio de 2005; aceptado el 5 abril de 2006.

Diánoia, vol. LI, no. 57 (noviembre 2006). 